\title{
Identification and Characterization of Reconstituted $\alpha$-Synuclein, Amyloid- $\beta$ and Tau Fibrils by Immunogold Negative Staining Electron Microscopy
}

\author{
Mike Reichelt ${ }^{1}$, Travis W. Bainbridge ${ }^{2}$, Racquel Corpuz ${ }^{2}$, Seung H. Lee ${ }^{3}$, James A. Ernst ${ }^{2}$, \\ Oded Foreman ${ }^{1}$, Meredith Sagolla ${ }^{1}$, Jasvinder K. Atwal ${ }^{3}$ and Gai Ayalon ${ }^{3}$ \\ 1. Department of Pathology, Genentech, South San Francisco, USA. \\ 2. Department of Protein Sciences, Genentech, South San Francisco, USA. \\ 3. Department of Neuroscience, Genentech, South San Francisco, USA.
}

Neurodegenerative diseases are a major public health concern in the aging population worldwide. Clinically, neurodegeneration manifests as cognitive decline and pathologically by dysfunction and loss of synapses, aggregation of misfolded proteins and progressive neuronal cell loss. The two most common neurodegenerative diseases are Alzheimer's disease, characterized by accumulation of betaamyloid plaques and neurofibrillary tau tangles, and Parkinson's disease, characterized by Lewy Bodies comprised of the protein alpha synuclein. In Alzheimer's disease and Parkinson's disease, the anatomical distribution and burden of tau tangles and synuclein Lewy bodies, respectively, correlate with progression of disease symptoms. These higher order structures of aggregated proteins are believed to originate with misfolded protein monomers that assemble into low molecular weight oligomers, which eventually form protein fibrils [1].

Electron microscopy (EM) is a valuable research tool in the investigation of the biochemistry, structure and kinetics of protein fibril formation in vitro, as well as in the morphological characterization of pathological protein deposits in cells and brain tissues in vivo. In order to support research efforts aimed at better understanding Alzheimer and Parkinson's disease pathology, we developed immunogoldlabeling protocols for the specific identification and characterization of fibrils generated in vitro from synthetic amyloid- $\beta$ peptide, recombinant $\alpha$-synuclein and recombinant Tau protein, respectively. Immunogold-labeling in combination with negative staining EM enables the simultaneous morphological characterization of pathogenic protein fibrils at high resolution with the precise antibodybased identification of these protein structures of interest.

Amyloid- $\beta$, was reconstituted from lyophilized chemically synthesized peptide [2]. $\alpha$-Synuclein and Tau fibrils were isolated from $E$. coli engineered to express the human protein. Fibrils were prepared using standard protocols $[3,4]$. For EM investigation, the protein solution was adsorbed to the surface of EM grids. The grid surface was then incubated with blocking solution to prevent any unspecific binding of reagent antibodies. Fibrils were labeled with primary antibodies specific for amyloid- $\beta, \alpha$-synuclein and Tau protein, respectively. Immunogold reagents were either biotinylated secondary antibodies in combination with streptavidin conjugated to $15 \mathrm{~nm}$ colloidal gold particles; or species specific Fabfragments directly conjugated with $10 \mathrm{~nm}$ colloidal gold. We performed rigorous negative controls to ensure the specific detection of the protein fibrils of interest. To control for unspecific adsorbtion of antibodies to the grid surface we employed "empty grid" controls (no adsorbed protein fibrils) in combination with all primary and secondary reagents. To test for specific antibody binding we used "unrelated antibody" controls (anti-gD antibody) over the protein fibrils of interest. In addition we controlled for potential cross-reactivity of the primary antibodies over "unrelated substrates", e.g. antisynuclein labeling was tested over both amyloid- $\beta$ and Tau fibrils. All samples were imaged with a JEOL JEM 1400 TEM at magnification from 5000x to 50000x using a GATAN Ultrascan 1000 camera. 
Analysis of labeled samples in the EM demonstrated that the immunogold protocols were both specific to the fiber type and sensitive. Individual Amyloid- $\beta, \alpha$-synuclein and Tau fibrils, respectively, were labeled with several gold particles each (sensitivity); and the ratio of specific gold particles to background was at least 100-fold (specificity). Using these protocols, it was possible to identify and distinguish various higher-order assembly stages of the proteins of interest. For instance, in $\alpha$-synuclein samples individual fibrils, clusters of fibrils and large amorphous aggregates were readily identified. These immunogold EM protocols will be valuable in the quality control (QC) of reconstituted fibrils, in evaluating the dynamics of fibril formation and aggregation and in the QC of new antibody reagents or drug candidates.
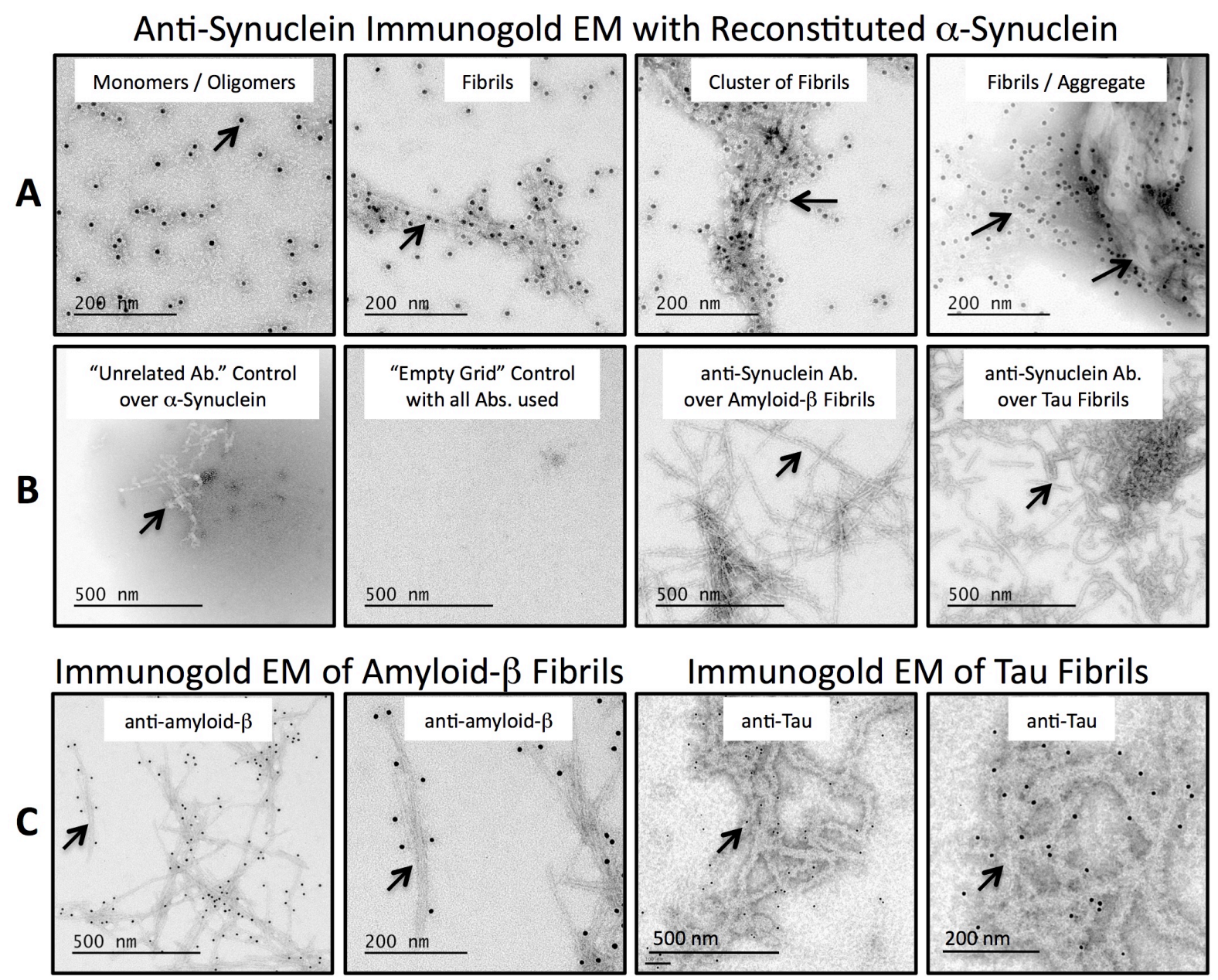

Figure 1. Specific immunogold EM detection of various assembly forms of $\alpha$-Synuclein (panel A) and of fibrils of Amyloid- $\beta$ (panel $\mathrm{C}$, left) or Tau protein (panel C, right). Examples of negative controls that were used to evaluate the specific binding of an anti-synuclein antibody are shown in panel B.

\section{References:}

[1] I Benilova, E Karran and BD Strooper, Nature Neuroscience 15 (2012), p.349.

[2] M Ultsch et al., Sci Rep 6 (2016), p.39374.

[3] SH Lee et al., Cell Rep 16:6 (2016), p.1690.

[4] Luk KC et al., Proc Natl Acad Sci 106 (2009), p.20051. 